\title{
Tolerance Analysis of Planar Mechanisms Based on a Residual Approach: A Complementary Method to DLM
}

\author{
Hector A. Tinoco $\mathbb{D D}^{1,2}$ and Sebastián Durango $\mathbb{D i}^{3}$ \\ ${ }^{1}$ Experimental and Computational Mechanics Laboratory, Universidad Autónoma de Manizales, Antigua Estación del Ferrocarril, \\ 170001 Manizales, Colombia \\ ${ }^{2}$ Institute of Physics of Materials, Czech Academy of Sciences, Žižkova 22, 61662 Brno, Czech Republic \\ ${ }^{3}$ Grupo Diseño Mecánico y Desarrollo Industrial, Universidad Autónoma de Manizales, Antigua estación del Ferrocarril, \\ 170001 Manizales, Colombia \\ Correspondence should be addressed to Hector A. Tinoco; htinoco@autonoma.edu.co
}

Received 20 January 2019; Revised 4 April 2019; Accepted 11 April 2019; Published 5 May 2019

Academic Editor: Ramon Sancibrian

Copyright (C) 2019 Hector A. Tinoco and Sebastián Durango. This is an open access article distributed under the Creative Commons Attribution License, which permits unrestricted use, distribution, and reproduction in any medium, provided the original work is properly cited.

\begin{abstract}
The kinematic performance of mechanisms is affected by different uncertainty sources involved in the manufacturing and assembling cycle; among these are the geometric variations. It is known that the effects of these variations produce position errors which are not usually included in the design process. With this objective, a complementary method for the tolerance analysis of planar mechanisms that incorporate geometric variations is presented in this paper. The approach is based on Direct Linearization Method (DLM) that does not consider all the kinematically admissible solutions. DLM naturally minimizes a residual functional $H$; however it is possible to maximize the residual by means of a proposed complementary method called H-Based Residual Method $(\mathrm{RMH})$. From the proposed methodology, local and global error domains can be defined to predict the maximum and minimum position errors caused by the input variations. DLM and RMH were applied in a four-bar mechanism with dimensional and angular variations to estimate positioning errors. The results show intervals where output positions were invariant with respect to angular variations of the crank. These computations were performed through a distance ratio established with the output deviations determined with nominal angular variations. Furthermore, domain errors were predicted for a set of positions generated by a multivariate normal random algorithm with 1000 combinations of input variations (links lengths). These domains delimited all solutions created in each position stage. It means that by applying the proposed methodology it is possible to estimate the geometric errors of any combination of variations.
\end{abstract}

\section{Introduction}

Positioning errors in mechanisms are generated by different uncertainty sources related to manufacturing, assembly, design, among others [1-3]. These uncertainties can be expressed as geometric variations that play a key role in the design process, since manufacturing costs are function of these variations (manufacturing costs with tight tolerances are greater; on the other hand, greater tolerances could make an assembly unfeasible with cheaper manufacturing [4]). In mechanisms, geometric variations can be considered as small defects in the link lengths or in the angular position of the joints. According to standards ANSI Y14.5-2018 [5] and ISO 1101 [6], tolerances and variations are defined as the allowable limits that are inherent in manufacturing and assembly processes. This means that geometrical variations are partially controllable parameters (tolerance) and in those cases the effects of small variations influence the kinematic performance of the mechanisms. Therefore, appropriate analysis tools should be developed to assess the kinematics of mechanisms including the different types of variations, as mentioned by Chase et al. [7] years ago. Their work analyzed different tolerance analysis with applications to the mechanism and machine design; this shows that the development of new tools to predict the effects of all type of variations is not a new challenge. 
The effects of small variations (link lengths and joint positions) on the kinematic performance can be assessed in early stages of the mechanism design process, which corresponds to the need for improving the tolerance analysis. LaraMolina et al. [8] present a study on planar 5R symmetrical mechanisms in which Monte Carlo simulation was used to determine the effects of small deviations of link lengths and joint clearances in several design atlases (Maximum Inscribed Workspace, Global Conditioning Index, and Minimum Conditioning Index). Jawale et al. [9] revise the use of a Partial Derivative Formulation (PDA) to estimate the output (angular) error in four-bar mechanisms (FBM) subjected to link tolerances. Their works validate that in the diversity of the planar mechanisms, most of the designs include FBM by its simplicity and functionality which shows that studying FBM is a current topic. Further, several error definitions were presented by Jawale et al. [9] (maximum error, RMS error, and total error) and the results were verified by means of a geometrical approach. The PDA allows isolating the effect of each link tolerance on each error category showing a significative difference between the total error (which is correlated to the geometric parameters) and the overestimated maximum error. Consequently, other analytic studies of the error are available, e.g., Hofmeister et al. [10] show a model that assumes known joint clearances and estimates the error by transforming to the kinematic image space. A FBM, cognate mechanisms, and multiloop mechanisms were analyzed. Flores [4] calculates the relation between variations in the dimensional parameters and the variation in the generalized coordinates (position error) by deriving a sensitivity matrix from kinematic constraints. Worst case scenario and statistical considerations were reported. The Direct Linearization Method (DLM) is recurrent in tolerance analysis, being $[7,11]$ seminal works. DLM has been advanced by several authors; e.g., Wittwer et al. [12] consolidated a work based on the estimation of the individual contribution of small variations of geometric parameters on assembly variables (dependent variables which guarantee the fulfillment of the kinematic constraints). To assess the contributions with DLM, a first-order Taylor's series expansion is used to linearize the variations of the kinematic constraints that represents a linkage. The method is usually expressed by a formulation of Jacobian matrices which allows deterministic and probabilistic error assessments, e.g., worst case error and root-sum-square error. DLM has been applied in different mechanism types by $[13,14]$, from rigid to flexible-body. Their work described how the coupler path of a four-bar mechanisms is affected (shifting in variance and in covariance of positioning errors) and unveiling critical parameters with highest contributions to the variations of assembly specifications.

Tolerance analysis in mechanisms and machines remains current; e.g., Steering and suspension systems of automobiles were analyzed by Kim et al. [15], Wang et al. [16], and Malvezzi and Hess-Coelho [17]. Kim et al. [15] studied the effects of accumulated geometric tolerances on wheel alignment by means of Monte Carlo simulations (probabilistic method). Modelling of high reliable steering systems according to the Ackerman's principle was assessed by Wang et al. [16]. Given the complexity of the kinematic structure of the steering mechanism, a probabilistic model based on a global surrogate model (build from a set of local surrogate models) was used to predict the maximal kinematic error of the system. The assessment of kinematic errors in the design of an active rear suspension was based on a three-degrees-of-freedom parallel mechanism, as presented by Malvezzi and Hess-Coelho [17]. Two error models were evaluated: the first one is based on direct kinematics (an analytic model) that allows the mapping of links tolerances and actuator inaccuracies to the wheel workspace; and the second one is based on a parametric optimization in which the objective function is the wheel alignment. Common to Kim et al. [15], Wang et al. [16], and Malvezzi and Hess-Coelho [17], is the development of analysis tools for the design of safer automotive systems, in the kinematic sense.

Tolerance analysis applied to transmissions has been considered by Armillotta [18] and Lin et al. [19]. An analogy from static analysis to tolerance analysis (analytic model) have been presented by [18], in which external forces correspond to assembly-level errors and calculated forces (reactions, internal forces which guarantee equilibrium) correspond to the sensitivity of the gearing part tolerances on the total error. Kinematic error and tolerance analysis of cycloidal transmissions were performed by Monte Carlo simulations in [19], by mapping geometric and manufacturing parameters to the transmission performance through gearing theory. Resulting kinematic error distributions allows optimizing the tolerance of the geometric parameters to minimize manufacturing costs with reliable gearing performance. Both works, Armillotta [18] and Lin et al. [19], are valuable design tools for transmissions with a required kinematic error performance.

Kinematic error and tolerance analysis in mechanisms, parallel robots, and structures have been developed recently by several authors. For example, Rameau et al. [20] assess the calculation of joint clearances necessary to guarantee the mobility in overconstrained planar and spatial mechanisms subjected to errors in the geometric parameters. The method is based on a geometrical model that allows estimating manufacturing parameters for mass production of the overconstrained mechanisms. The evaluation of the assembly precision in planar mechanisms is presented by Zhao et al. [21]. The error prediction is conducted by a three-step assembly algorithm and each step has associated a particular error model, with which was assessed the assembly precision. Different from Rameau et al. [20], the three-step algorithm is intended for the evaluation of static structures (interpreted as overconstrained mechanisms). Fan et al. [22] present a kinematic calibration of a multiaxis machine tool based on geometric errors of the guideways. The calibration performed mapping measured kinematic errors to the geometric profile of the guideways. The fitted error model allows predicting kinematic errors (position and orientation) in the workspace of the machine tool; then it is possible to modify the manufacturing code (e.g., G-code) to minimize the kinematic errors. Design of a parallel robot, with full-circle rotation, based on error modeling and tolerance analysis is developed in $\mathrm{Ni}$ et al. [23]. Tolerances mapping were assessed through a kinematic model to perform a sensitivity analysis of the 
end moving platform with respect to the geometric errors. The sensitivity analysis was used as a tolerance design tool by means of nonlinear constrained optimization. Common to Rameau et al. [20], Zhao et al. [21], Fan et al. [22], and Ni et al. [23] is the use of tolerance analysis as a keystone in the design process of mechanisms and machines with required performance (to guarantee mobility and assembly and to minimize positioning errors in machine tools and parallel robots).

The estimation of individual contributions of geometrical variations to the positioning error of mechanisms, by either probabilistic or deterministic methods, it is a common place in the aforementioned works. This work presents a methodology to predict position errors in planar mechanisms with geometric variations (tolerance analysis). Jawale et al. [9] and Wang et al. [16] recognized the importance of improving DLM (when comparing it with respect to Monte Carlo simulations) and find practical limits (possible subestimation of maximum normal-to-path errors). Therefore, a complementary method to DLM is developed and applied in this study. The methodology is based on the minimization and maximization of a functional obtained from numerical residuals that generate the variations in the closed loop vector analysis. Error domains are designed from the deviations to determine the positional variation as a deterministic or probabilistic solution.

\section{Theoretical Background}

2.1. Kinematics of Planar Mechanisms. Let us consider a $n$-bar mechanism composed by one loop which is represented by a closed vector loop as shown in Figure 1(a). For a mechanism of $m$-loops, the same principles can be applied that will be exposed for one loop mechanisms [24]. For describing the kinematics of planar mechanisms, a vector notation is used in a closed loop such that for one loop its mathematical representation is given by

$$
h=\sum_{j=1}^{n-1} r_{j} e^{i \theta_{j}}-r_{n} e^{i \theta_{n}}=0,
$$

where $\vec{r}_{j}=r_{j} e^{i \theta_{j}}, \forall j=1,2, \ldots, n$. Applying Euler's identity on (1), we have two projections on a coordinate system as follows:

$$
h_{x}=\sum_{j=1}^{n-1} r_{j} \cos \left(\theta_{j}\right)-r_{n} \cos \left(\theta_{n}\right)=0
$$

and

$$
h_{y}=\sum_{j=1}^{n-1} r_{j} \sin \left(\theta_{j}\right)-r_{n} \sin \left(\theta_{n}\right)=0 .
$$

By organizing (2) and (3), a system of equations is established in the following way:

$$
\mathbf{A}_{0} \mathrm{X}-\mathbf{B}_{0} \mathrm{U}=0
$$

Equations (2) and (3) define two sets of variables: an independent set $\mathrm{X}=\left\{q_{j} \mid k, n \subset j\right\}$ and a dependent set $\mathrm{U}=\left\{x_{i} \mid p, r \subset i\right\}$. The dependent set is a function of kinematic variables and geometrical parameters (lengths $q_{k}, x_{p}$ and angles $q_{n}, x_{r}$ ). Equation (4) describes the kinematics of a mechanism without variations, where $\mathbf{A}_{\mathbf{0}}$ and $\mathbf{B}_{\mathbf{0}}$ are composed matrices by known parameters. The solution $U$ determines the nominal values that satisfy the kinematics for given inputs $\mathrm{X}$.

2.2. Direct Linearization Method (DLM). Direct Linearization Method (DLM) is a method established for solving the kinematics of mechanisms with geometric variations (dimensional and angular variations). DLM was initially proposed by Marler [11] and it is based on approximating variational parameters of closed vector loops by means of a linearized functional through Taylor's series expansion as explained in $[14,25]$. The approximations are obtained from nominal values $\mathrm{Y}=\{\mathrm{X}, \mathrm{U}\}$ with the aim of determining the variations $\mathrm{dU}=\left\{d x_{i} \mid p, r \subset i\right\}$ that depend on known parameters $\mathrm{dX}=\left\{d q_{j} \mid k, n \subset j\right\}$. Before applying DLM, the solutions $q_{j}$ should be solved as well as the set of expected variations $d q_{j}$. Taking into account the following considerations, a variational closed vector loop is defined for the mechanism represented in Figure 1(b): Let's consider a functional $H(\mathbf{Y}+\mathbf{d Y})$ linearized about $h$ by using Taylor's series expansion truncated in the second term; therefore, it is determined that

$$
H_{x}=h_{x}+\sum_{j=1}^{k+n} \frac{\partial h_{x}}{\partial q_{j}} d q_{j}+\sum_{i=1}^{p+r} \frac{\partial h_{x}}{\partial x_{i}} d x_{i}=0,
$$

and

$$
H_{y}=h_{y}+\sum_{j=1}^{k+n} \frac{\partial h_{y}}{\partial q_{j}} d q_{j}+\sum_{i=1}^{p+r} \frac{\partial h_{y}}{\partial x_{i}} d x_{i}=0 .
$$

As established in (2) and (3), it is known that $h_{x}=$ $h_{y}=0$ (obtained from nominal values) since both solutions represent a closed vector loop. If (5) and (6) are organized, a set of equations is written as a system of equations such as

$$
\mathbf{A d X}+\mathbf{B d U}=0
$$

being

$$
\begin{aligned}
& \mathbf{A}=\left[\begin{array}{llll}
\frac{\partial h_{x}}{\partial q_{1}} & \frac{\partial h_{x}}{\partial q_{2}} & \cdots & \frac{\partial h_{x}}{\partial q_{k+n}} \\
\frac{\partial h_{y}}{\partial q_{1}} & \frac{\partial h_{y}}{\partial q_{2}} & \cdots & \frac{\partial h_{y}}{\partial q_{k+n}}
\end{array}\right] \\
& \mathbf{B}=\left[\begin{array}{lll}
\frac{\partial h_{x}}{\partial x_{1}} & \cdots & \frac{\partial h_{x}}{\partial x_{p+r}} \\
\frac{\partial h_{y}}{\partial x_{1}} & \cdots & \frac{\partial h_{t}}{\partial x_{p+r}}
\end{array}\right]
\end{aligned}
$$

where $\mathrm{dX}=\left[\begin{array}{llll}d q_{1} & d q_{2} & \cdots & d q_{k+n}\end{array}\right]^{T}$ and $\mathrm{dU}=$ $\left[\begin{array}{lll}d x_{1} & \cdots & d x_{p+r}\end{array}\right]^{T}$. A and $\mathbf{B}$ are Jacobian matrices determined 

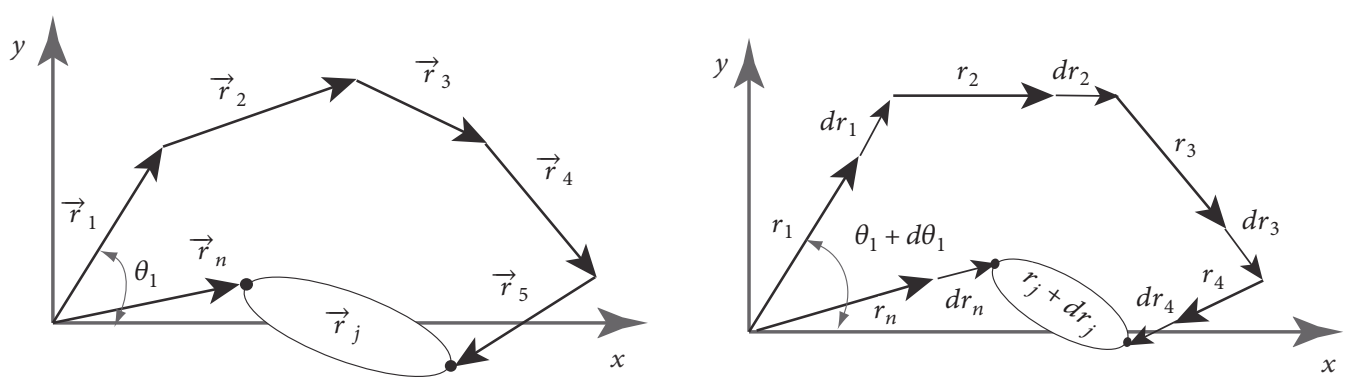

$r_{j} \angle \theta_{j}, \forall_{j}=1,2,3, \ldots, n$

(a) (b)

FIGURE 1: General vector loop: (a) without variations; (b) with variations.

of (2) and (3). A solution is obtained from (7); on this basis it can be calculated that

$$
\mathrm{dU}=-\mathbf{B}^{-1} \mathbf{A d X}=\mathbf{S d X}
$$

where $\mathbf{S}$ is a sensitivity matrix as mentioned by Leishman et al. [14]. All basic principles about calculations for DLM can be reviewed in references $[12,13,25]$. For velocity and acceleration calculations, Leishman et al. [14] showed in detail the procedure, which is based on the differentiation of the position equations without and with variations.

2.3. H-Based Residual Method (RMH). In this section $H$ Based Residual Method (RMH) is presented as an alternative method to solve the variations dU; it considers $H(\mathbf{Y}+\mathbf{d Y})$ as a residual functional since this is determined by numerical approximations DLM. The proposition of the method is based on the propagation of the numerical errors that are generated on $H(\mathbf{Y}+\mathbf{d Y})$ by the truncated Taylor's series. It means that there are residual errors. Basically, the solution that will be presented is complementary to DLM. Therefore, the following aspect is analyzed: when $H(\mathbf{Y}+\mathbf{d Y})=0$, the found solutions are those that minimize the residuals $H_{x}$ and
$H_{y}$, such as it is carried out in DLM. However, there are kinematically admissible solutions in which the errors can maximize $\partial H(\mathbf{Y}+\mathbf{d Y}) / \partial \mathrm{Y}=0$. It implies that some kinematic solutions can propagate errors higher than DLM. The above statement can be verified from the sensitivity of each variation in $q_{j}$ and $x_{i}$ with respect to $H$. Then, the statement is applied on (5) and (6) to determine maximum or minimum values on $H$ in the following way: at $x$-direction it is calculated as

$$
\sum_{j=1}^{n+k} \frac{\partial H_{x}}{\partial q_{j}}+\sum_{i=1}^{r+p} \frac{\partial H_{x}}{\partial x_{i}}=0
$$

and at $y$-direction, it is determined that

$$
\sum_{j=1}^{n+k} \frac{\partial H_{y}}{\partial q_{j}}+\sum_{j=1}^{r+p} \frac{\partial H_{y}}{\partial x_{i}}=0
$$

From (10) and (11), the following system of equations is established as follows:

$$
\mathrm{dU}=-\mathbf{B}_{\mathbf{s}}^{-1} \mathbf{A}_{\mathrm{s}} \mathrm{dX}=\mathbf{S}_{\mathrm{s}} \mathrm{dX},
$$

where and $S_{s}$ is a sensitivity matrix of second order. Equation (12) is similar to (9) in its structure; but matrices $\mathbf{A}_{s}$ and $\mathbf{B}_{\mathrm{s}}$ have different meanings.
2.4. Geometric Variations $d U$ from Eigenvalues. In this section is shown how variations can be determined from eigenvalues obtained from Jacobian matrices; these solutions help 
to define an error domain in which the output position should exist inside it. Given that both methods (Sections 2.2 and 2.3) show a similar equation to solve $\mathrm{dU}$, it is possible to compute a solution from the variations of the output parameters such that the following eigenvalue problem can be written using (7) or (12), as follows:

$$
-\mathbf{A d X}=\mathrm{BdU}=\lambda I \mathrm{dU}
$$

and then, we know that

$$
|\mathbf{B}-\lambda I| \mathrm{dU}=0
$$

where $\lambda$ represents the eigenvalues of the sensitivity matrix B; after determining $\lambda_{k}, k=1,2$, we can obtain the following solutions applying (14) as

$$
\mathrm{dU}_{\mathrm{eig}}=-\frac{\mathrm{AdX}}{\lambda_{k}}, \quad \forall k=1,2 .
$$

To determine maximum variations $\mathrm{dU}_{\max }=\mathrm{d} \mathrm{U}_{\text {eig }}$, these should be obtained with $\lambda_{\min }=\min \left\{\lambda_{1}, \lambda_{2}\right\}$. It was explained before that $\mathrm{dU}$ can be determined from any method, for example, RMH and DLM, and that there exist two solutions for each one, those determined from the nominal extremes of the input variations $d q_{k}^{+}$and $d q_{k}^{-}$. So, $\mathrm{dU}_{\max }^{+}$and $d U_{\max }^{-}$are determined from the eigenvalues solution.

\section{Position Prediction}

3.1. Residual Approach for $h$. It was discussed in Section 2 that by definition $h=0$, which satisfies a closed vector loop without variations. However, if the variations are included in the mechanism; the obtained solutions generate a numerical error or residual on $h\left(q_{j}+d q_{j}, x_{i}+d x_{i}\right)=\bar{e}, \forall j=1,2, \ldots, n$, caused by the approximation determined for dU (DLM and $\mathrm{RMH}$ methods). In Figure 2, a scheme of the propagated error by the approximation $\mathrm{dU}$ is shown. The numerical error is considered as a vector; it denotes that $h\left(q_{k}+d q_{k}, x_{p}+\right.$ $\left.d x_{p}\right)-\bar{e}=0$ should satisfy the equality. The value of $\bar{e}$ can be estimated with admissible solutions determined from the following cases if $d q_{k}^{+}$and $d q_{k}^{-}$. Let us consider that $d q_{k}$ is a known parameter of input (variation) which has a nominal value defined before solving $\mathrm{dU}$. Then, for $d q_{k}^{+}>0$ and $d q_{k}^{-}<$ 0 , two different solutions are achieved for dU. Each solution generates a residual such that for $d q_{k}^{+}$there are determined $h_{x}^{+}$and $h_{y}^{+}$that represent the projections of the directional residuals on both real and imaginary axes. Analogously, for $d q_{k}^{-}$, there are computed $h_{x}^{-}$and $h_{y}^{-}$.

Considering the directional residuals for each case of variations $\left(d q_{k}^{-}\right.$and $\left.d q_{k}^{+}\right)$, the Euclidean norm of the residuals is calculated as.

$$
\begin{array}{r}
\bar{e}^{-}=\sqrt{\left(h_{x}^{-}\right)^{2}+\left(h_{y}^{-}\right)^{2}}, \quad \text { if } \exists d q_{k}^{-}<0 \\
\text { and } \bar{e}^{+}=\sqrt{\left(h_{x}^{+}\right)^{2}+\left(h_{y}^{+}\right)^{2}}, \quad \text { if } \exists d q_{k}^{+}>0 .
\end{array}
$$

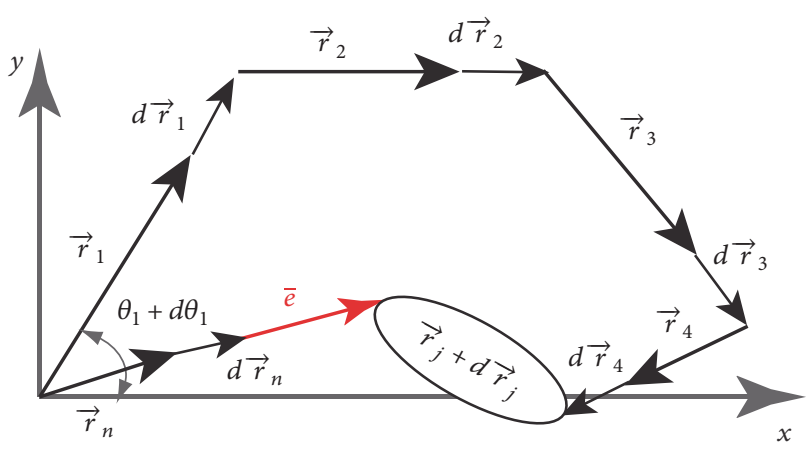

FIGURE 2: Error propagation over the general vector loop with variations.

To maximize the propagated error by the solutions $\mathrm{dU}$, a normalization is proposed as follows:

$$
\bar{e}=\sqrt{\left(\bar{e}^{-}\right)^{2}+\left(\bar{e}^{+}\right)^{2}}
$$

Equation (18) can be defined as the maximum propagation of the error produced by the approximations. In the case of DLM the solution $\mathrm{dU}$ generates a residual of $\bar{e} \approx 0$, due to the nature of the solution. A discussion will be performed in the next sections. The principal purpose of the error propagation is to extend an error domain from the estimated positions with $\mathrm{dU}$ which are kinematically admissible, as for example those solutions obtained with RMH and DLM.

3.2. Position Prediction in a Local Error Domain for Input Variations. According to the traditional mechanism design, the output positions are deterministically predicted. However, when the geometry of the mechanisms varies by different circumstances, the output positions should exist inside an error domain which can be seen as a tolerance as proposed by different studies $[13,26,27]$. With this purpose, a solution space is established and delimited by five points that belong to the output positions of a mechanism with fixed variations $\mathrm{dX}$. It means that geometric variations are known. Those variations are chosen as steady parameters since the variation source will be taken only from the input variable $\left(q_{k}\right)$. This presents a range of variation given by $d q_{k} \in\left(d q_{k}^{-}, d q_{k}^{+}\right)$. Each estimated output position will be specified in the following way; $p_{k}$ is the position without including variations in the input, $p_{k}^{+}$is the position when $\exists d q_{k}^{+}$and calculated with $\mathrm{dU}^{+} \cdot p_{k}^{-}$is the position when $\exists d q_{k}^{-}$and calculated with $\mathrm{dU}^{-}$. $p_{k}^{\lambda+}$ and $p_{k}^{\lambda-}$ are the positions obtained from the eigenvalues when $\exists d q_{k}^{+}$and $d q_{k}^{-}$with $\mathrm{dU}_{\max }^{+}$and $\mathrm{dU}_{\max }^{-}$. The five points are depicted in Figure 3(a). Hence, an error domain $\Omega_{k}$ for the output positions is defined as a place specified by the boundary of a circumference $\Gamma_{k}$ with an established center in

$$
\bar{p}_{k}=\frac{1}{3}\left(p_{k}+p_{\delta(k)}\right)
$$

where $p_{\delta(k)}=\left(p_{k}^{+}+p_{k}^{-}\right) / 2$. Equation (19) defines a prediction that represents the closest distance. However, an 


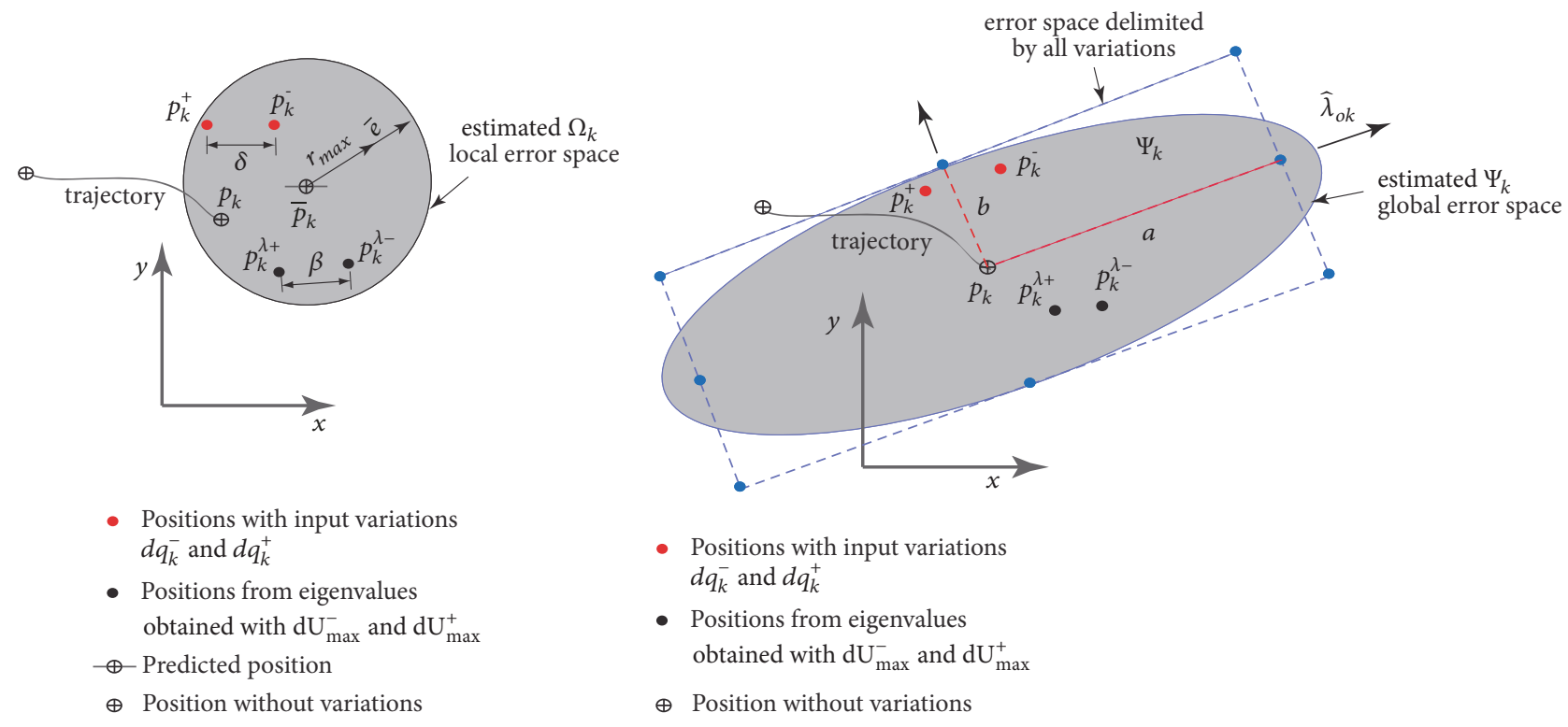

(a)

(b)

Figure 3: (a) Local domain error. (b) Global domain error.

error domain is defined by the radius $\Gamma_{k}$ determined by the maximum distance as

$$
r_{\max (k)}=\max \left|\bar{p}_{k}-\left\{p_{k}, p_{k}^{+}, p_{k}^{-}, p_{k}^{\lambda+}, p_{k}^{\lambda-}\right\}\right|+\bar{e}_{k},
$$

where $k$ indicates any position. The circle area generates a local domain error $\Omega_{k}$ that includes the residual $\bar{e}_{k}$ that remains after approximating the output position as illustrated in Figure 3(a). The five points mentioned above are depicted also in the figure. The distance between $p_{k}^{-}$and $p_{k}^{+}$is described as $\delta$. According to $\delta$, it is possible to say that the distance is minimum in regions where the errors in the positions are invariant to $d q_{k}^{+}$and $d q_{k}^{-}$. Further, we can point out that when $r_{\max (k)}$ is minimum, it indicates where the mechanism will be more accurate.

To complement the theoretical analysis, we propose the distance $\beta$ established between $p_{k}^{\lambda+}$ and $p_{k}^{\lambda-}$ as a sensitive parameter. The following relation is suggested to determine when $p_{k}^{+} \approx p_{k}^{-}$through the expression

$$
\xi=\frac{\beta}{\delta}
$$

Equation (21) will permit examining intervals where the output positions $p_{k}^{+}$and $p_{k}^{-}$are invariant to the input variations imposed by $d q_{k}^{-}$and $d q_{k}^{+}$. It means that $\delta \approx 0$ when the mechanism can have any input variation $d q_{k} \in\left(d q_{k}^{-}, d q_{k}^{+}\right)$.

3.3. Position Prediction with a Global Error Domain for All Kinematic Variations. The main purpose of designing mechanisms is to convert a given input motion into a desired output motion. The accuracy of the motion is usually measured by the generated output deviations. These are produced by the geometric variations of its linkages or by angular variations provided by assembly tolerances. For mechanisms with geometric variations, there exist a set of solutions that define an error domain determined by all possible output positions related to each given combination of variations. It indicates that each position should be evaluated in all combinations of input variations with the aim to estimate the maximum error in each position state. However, this task can be expensive in computational terms since depending on the number of combinations, the number of solutions will define the computation time in each solution stage. In this way, a methodology to estimate a global error domain is presented which will represent all possible solutions marked in an error ellipse for each projected position. Therefore, let us consider a mechanism with nominal variations given as input parameters. An approximation can be constructed from the nominal variations to establish a global domain error $\Psi_{k}$; it is defined by an ellipse oriented in direction $\hat{\lambda}_{o k}$ as shown in Figure 3 (b). The unitary vector $\hat{\lambda}_{o k}$ is determined from the following expression:

$$
\hat{\lambda}_{o k}=\frac{p_{k}^{+}-p_{k}^{-}}{\left\|p_{k}^{+}-p_{k}^{-}\right\|} .
$$

The minor axis of the error ellipse is given by

$$
b_{k}=\sqrt{2}\left(p_{\delta(k)}-p_{k}+\bar{e}_{k}\right), \quad \forall k=1, \ldots, p,
$$

and the major axis is approximated as follows:

$$
a_{k}=3 \sqrt{2}\left(p_{\lambda(k)}-p_{k}+\bar{e}_{k}\right),
$$


where $p_{\lambda(k)}=\left(p_{k}^{\lambda+}+p_{k}^{\lambda-}\right) / 2$ and $p$ means number of positions. The error ellipse is a domain that defines the probability of each output position representing all possible combinations of variations of the mechanism.

\section{Case Study}

4.1. Four-Bar Mechanism (FBM). To evaluate the proposed method in Section 2, a planar Four-Bar Mechanism (FBM) is considered and shown in Figure 4(a). The fixed link is the number 1 , as illustrated in the figure. The angular orientations are labeled as $\theta_{j}$ and the lengths of the links are denoted by $r_{j}, \forall j=1,2,3,4$. The parameters $\theta_{k}, r_{j} ; \forall k=1,2$; are known; and $\mathrm{U}=\theta_{5-k}$ are the output dependent parameters.

To describe the position of the FBM, each link is represented by a vector as shown in Figure 4(b). The set of vectors is expressed as $\vec{r}_{i}\left(r_{i}, \theta_{i}\right)=r_{i} e^{j \theta_{i}}$; then closed vector loop is written as

$$
r_{1} e^{j \theta_{1}}+r_{2} e^{j \theta_{2}}+r_{3} e^{j \theta_{3}}-r_{4} e^{j \theta_{4}}=h=0 .
$$

Equation (25) represents a complete description of any geometric configuration for determining the positioning of the FBM. To include the variations in the FBM, the nominal vectors are extended by means of variational vectors as depicted in Figure 4(c). Applying the procedures of linearization exposed in Section 2.2, it is determined that

$$
\begin{aligned}
H= & h+\sum_{j=1}^{n-1} \frac{\partial h}{\partial \theta_{j}} d \theta_{j}+\sum_{j=1}^{n-1} \frac{\partial h}{\partial r_{j}} d r_{j} \\
& -\left(\frac{\partial h}{\partial r_{n}} d r_{n}+\frac{\partial h}{\partial \theta_{n}} d \theta_{n}\right),
\end{aligned}
$$

where $n=4$. Then, applying (26) on (25), we obtain the variational vector loop that is represented by

$$
\begin{aligned}
& r_{1} d \theta_{1} j e^{j \theta_{1}}+d r_{1} e^{j \theta_{1}}+r_{2} d \theta_{2} j e^{j \theta_{2}}+d r_{2} e^{j \theta_{2}} \\
& \quad+r_{3} d \theta_{3} j e^{j \theta_{3}}+d r_{3} e^{j \theta_{3}}-r_{4} d \theta_{4} j e^{j \theta_{4}}-d r_{4} e^{j \theta_{4}} \\
& =H .
\end{aligned}
$$

It is important to note that a particular case is obtained from (27) and it is given by $H=0$, with these solutions $H$ is minimized; it means that DLM is determined (see Section 2.2). To evaluate the FBM shown in Figure 4, in Table 1, the independent parameters are listed, corresponding to those proposed by Leishman et al. [14] to validate the DLM.

4.2. Solution $d U$ from DLM for the FBM. To describe the position of the FBM,

To obtain the solutions with DLM, those that determine the geometric variations, Jacobian matrices $\mathrm{A}$ and $\mathbf{B}$ are computed from (8) so it is obtained that

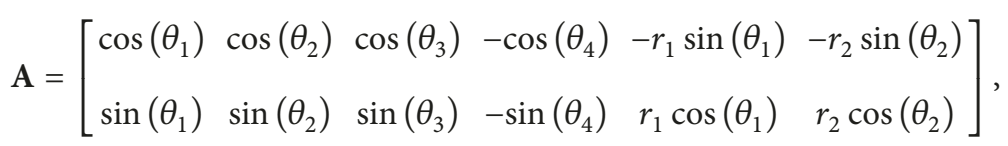

and

$$
\mathbf{B}=\left[\begin{array}{cc}
-r_{3} \sin \left(\theta_{3}\right) & r_{4} \sin \left(\theta_{4}\right) \\
r_{3} \cos \left(\theta_{3}\right) & -r_{4} \cos \left(\theta_{4}\right)
\end{array}\right] .
$$

The solution $d U$ is determined with (9), where $d U=$ $\left[\begin{array}{lll}d \theta_{3} & d \theta_{4}\end{array}\right]^{T}$ and $\mathrm{dX}=\left[\begin{array}{llllll}d r_{1} & d r_{2} & d r_{3} & d r_{4} & d \theta_{1} & d \theta_{2}\end{array}\right]^{T}$. It is known that $\mathbf{S}=-\mathbf{B}^{-1} \mathbf{A}$, where we can point out that $\mathbf{S}$ is called sensitivity matrix. In general terms, the solution presented in (9) is a deterministic solution since a value $\mathrm{dU}$ is obtained for each input variation. References $[12,14]$ estimated the kinematic variations $\mathrm{dU}$ by means of a statistic model based on the deterministic model; it is considered as worst case and it can be calculated by

$$
\mathrm{du}=\sqrt{\sum_{j=1}^{n=4}\left(\mathrm{~S}_{i j} \mathrm{dX}_{j}\right)^{2}}, \quad \forall i=1,2,
$$

where $\mathrm{dX}_{j}$ and $\mathrm{S}_{\mathrm{ij}}$ represent each element of $\mathrm{dX}$ and $\mathbf{S}$.

4.3. Solution $d U$ from $R M H$ for the FBM. To solve the variations $\mathrm{dU}$ from the sensitivity $H$ (residual functional), (10) and (11) are applied such that the following matrices are calculated

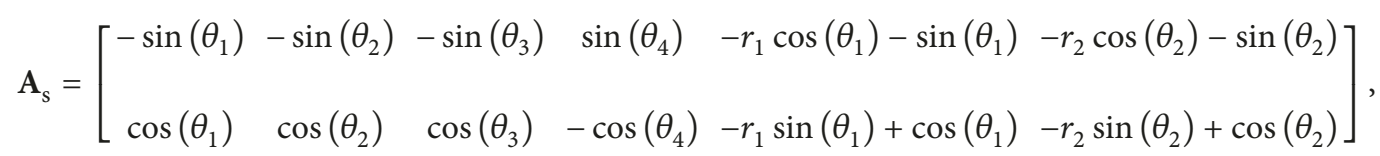




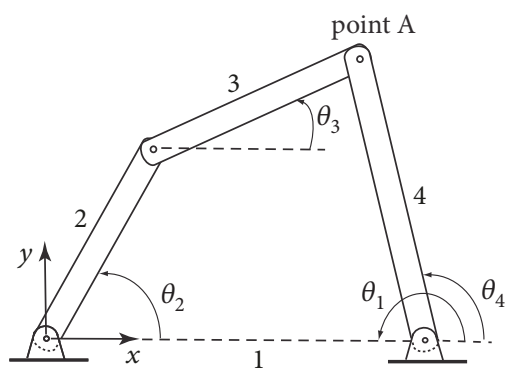

(a)

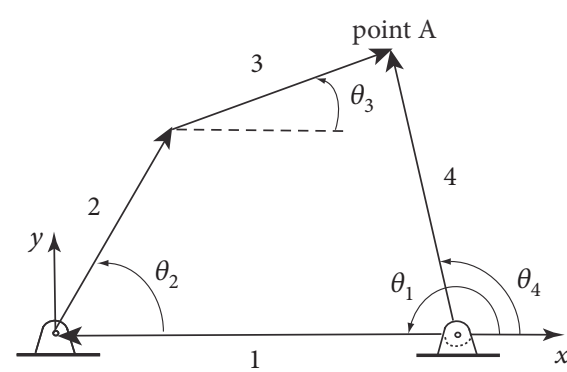

(b)

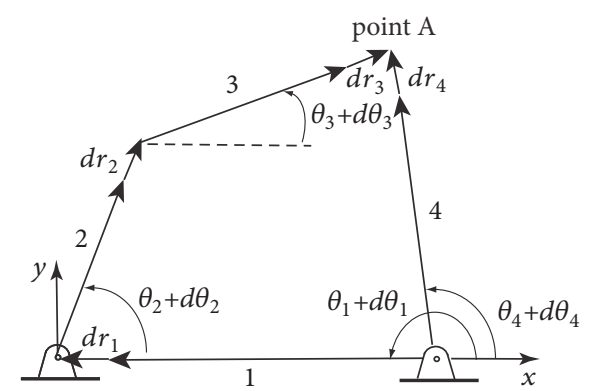

(c)

FIgURE 4: (a) FBM. (b) Vector loop without variations. (c) Vector loop with variations.

TABLE 1: Dimensions and kinematic variations for the FBM [14].

\begin{tabular}{lcc}
\hline Item & $\mathrm{X}[\mathrm{cm}]$ & Variation $\mathrm{dX}[\mathrm{cm}]$ \\
\hline$r_{1}$ & 5 & 0.02 \\
$r_{2}$ & 2 & 0.01 \\
$r_{3}$ & 5 & 0.02 \\
$r_{4}$ & 4.5 & 0.015 \\
$\theta_{1}$ & $\pi$ & 0 \\
$\theta_{2}$ & $0-2 \pi$ & $d \theta_{2} \in(-17,17) \times 10^{-4}$ \\
\hline
\end{tabular}

and the matrix

$$
\mathbf{B}_{\mathrm{s}}=\left[\begin{array}{ll}
-r_{3} \cos \left(\theta_{3}\right)-\sin \left(\theta_{3}\right) & r_{4} \cos \left(\theta_{4}\right)+\sin \left(\theta_{4}\right) \\
-r_{3} \sin \left(\theta_{3}\right)+\cos \left(\theta_{3}\right) & r_{4} \sin \left(\theta_{4}\right)-\cos \left(\theta_{4}\right)
\end{array}\right]
$$

knowing that $\mathrm{dU}=\mathbf{S}_{\mathrm{s}} \mathrm{dX}$ and $\mathbf{S}_{\mathbf{s}}=-\mathbf{B}_{\mathbf{s}}^{-1} \mathbf{A}_{\mathrm{s}}$.

\section{Results and Discussion for the FBM}

5.1. Comparison between DLM and RMH. In Figure 5, there are observed the obtained results for $d \theta_{3}$ and $d \theta_{4}$ considering the input variations on $\theta_{2}, d \theta_{2}^{ \pm}= \pm 1.7 \times 10^{-3} \mathrm{rad}\left(d q_{k}^{ \pm}\right)$ as listed in Table 1 . The results computed for $\mathrm{dU}$ with both methodologies DLM and RMH are compared. As explained in previous sections, $\mathrm{RMH}$ solutions are based on the sensitivity of the linear approximations performed for $H$ (present work, see Section 2.3) and DLM solutions are focused on the minimization of $H$ (see $[12,14])$. In general terms, it is seen that the results determined for $d \theta_{3}$ and $d \theta_{4}$ show a similar trend for both solution cases. However, it is important to mention that $d \theta_{3}$ and $d \theta_{4}$ evidence a different behavior for each input variation $d \theta_{2}^{ \pm}$. In the example proposed by Leishman et al. [14] only $d \theta_{2}^{+}$was taken into account.

Figure 5(a) shows that DLM solutions presented higher variations from 3 and $4.4 \mathrm{rad}$ for $d \theta_{4}$ and $d \theta_{3}$. On the other hand for $\mathrm{RMH}$, variations were higher in the rest of the intervals, approximately. This indicates that both methods can maximize and minimize the variations with different solutions that are kinematically admissible. Figure 5(b) shows that DLM presented maximum variations from 0 until 1 and $3 \mathrm{rad}$, for $d \theta_{4}$ and $d \theta_{3}$. Consequently, RMH presented maximum variations in the remaining part of the domain. It is important to point out that maximum output variations do not mean that position errors are higher for those intervals, since the position depends on the final configuration of the mechanism. It implies that the all solutions should satisfy that $h\left(q_{k}+d q_{k}, x_{p}+d x_{p}\right)=\bar{e} \approx 0$ (closed vector loop).

Given that all geometric parameters are known including its variations, it is verified that the solutions satisfy $h_{x}$ and $h_{y}$ by means of the propagated error by both methods DLM and $\mathrm{RMH}$, as explained in Section 3.1. In Figure 6 the correlations between $h_{x}$ and $h_{y}$ are shown for each input variation $d \theta_{2}^{+}$and $d \theta_{2}^{-}$. It is observed that errors determined by $\mathrm{RMH}$ are greater than errors calculated with DLM approximately $10^{2}$ times. These correlations are explained as the propagated errors influence the geometric configuration of the mechanism. The existence of the errors indicates that a confidence domain can be established to predict the output positions with both methodologies; as it will be explained in the next sections.

In Figure $7(\mathrm{a})$, the propagated errors determined from $h\left(q_{k}+d q_{k}, x_{p}+d x_{p}\right)=\bar{e}$, and calculated with (17) and (18) are shown. These were obtained with each input variation $d \theta_{2}^{-}$and $d \theta_{2}^{+}$. As proposed in Section 3.1, it is seen that the magnitude of $\bar{e}$ computed from DLM is much lower than the values calculated from RMH, as expected by the definition of the method. $\bar{e}$ is defined as a vector and represents the deviation of the geometric configuration of the mechanisms; it means that $\bar{e}$ complements the vector chain to close it.

5.2. Accuracy Errors in the Position by Input Angular Variations $\left(d \theta_{2}^{-}\right.$and $\left.d \theta_{2}^{+}\right)$. In this section, there are presented the computations for the output position errors generated by the input angular variations $d \theta_{2}^{-}$and $d \theta_{2}^{+}$applying both methods (RMH and DLM). To propose a discussion about the results, two concepts are defined: output position error and output accuracy. Output position error refers to the deviation that presents the predicted position with respect to the nominal output position $\left(p_{k}\right)$. Error accuracy means the deviation between output positions (distance among them $\delta$ ) obtained with different input variations, as for example, the extreme angular values $d \theta_{2}^{-}$and $d \theta_{2}^{+}$.

In Figure 8(a), the output position error is shown $\mid p_{\delta(k)}-$ $p_{k} \mid$ determined with the mean value $p_{\delta(k)}=\left(p_{k}^{+}+p_{k}^{-}\right) / 2$ (as described in Figure 3). It is noted that both methods 


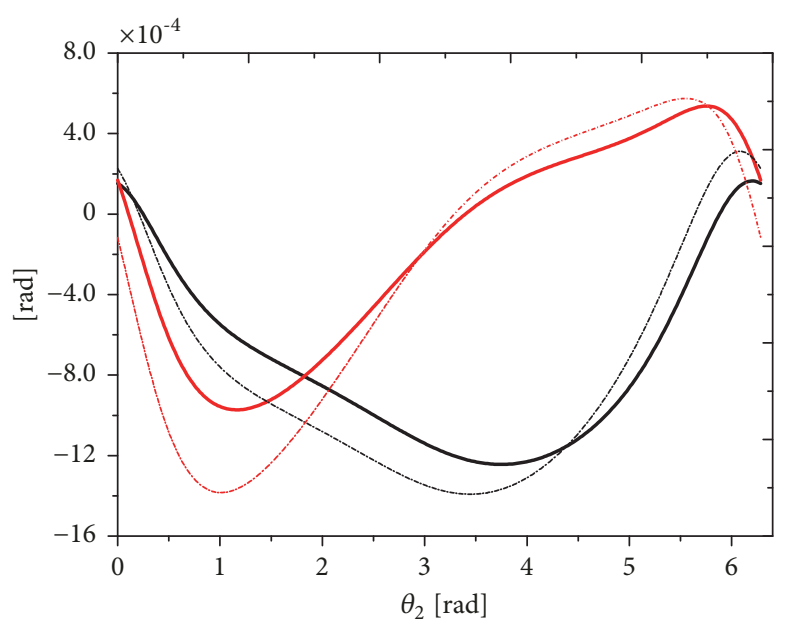

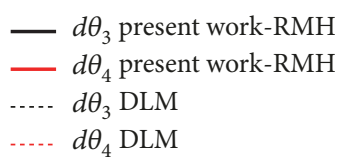

(a)

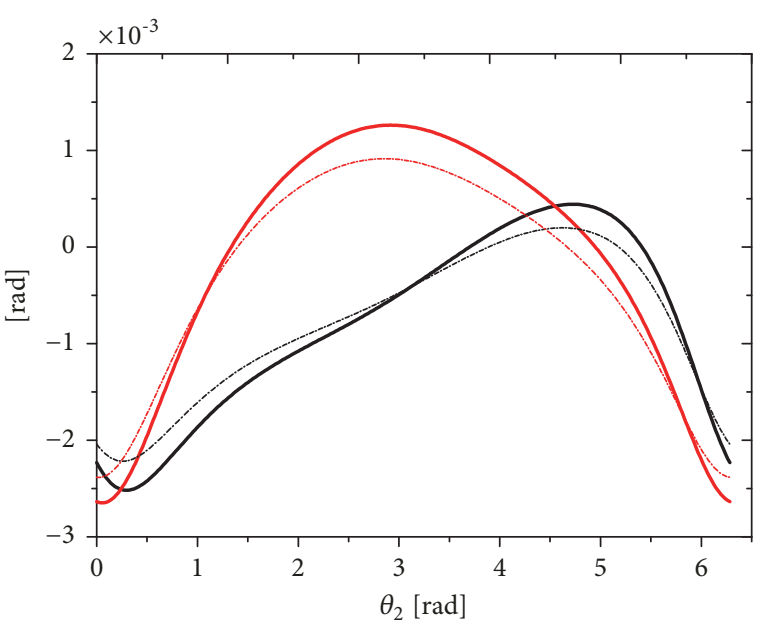

$\ldots d \theta_{3}$ present work-RMH
$\ldots d \theta_{4}$ present work-RMH
$\ldots . .-d \theta_{3}$ DLM
$\ldots . . . d \theta_{4}$ DLM

(b)

Figure 5: Solving dU for (a) $d \theta_{2}^{-}$(b) $d \theta_{2}^{+}$.

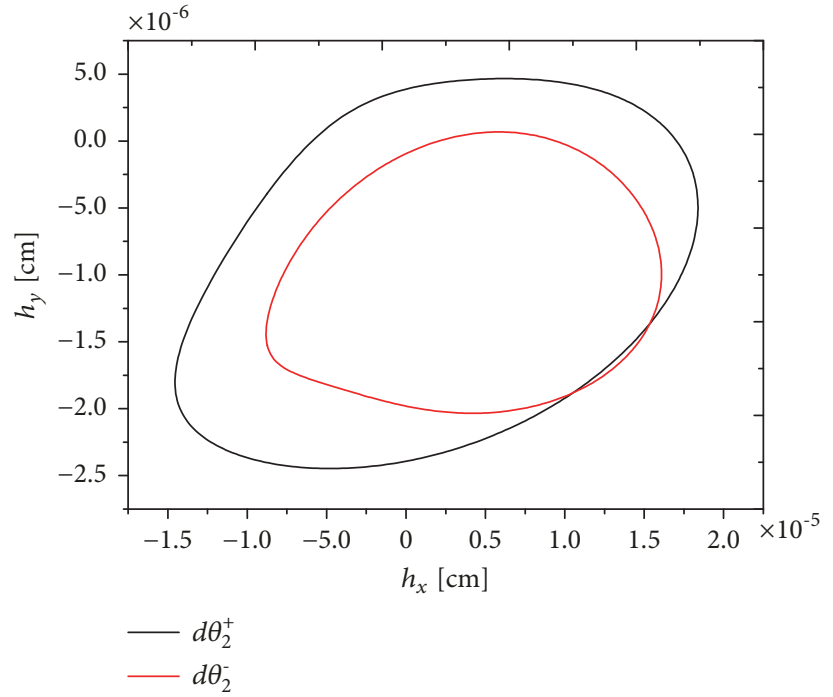

(a)

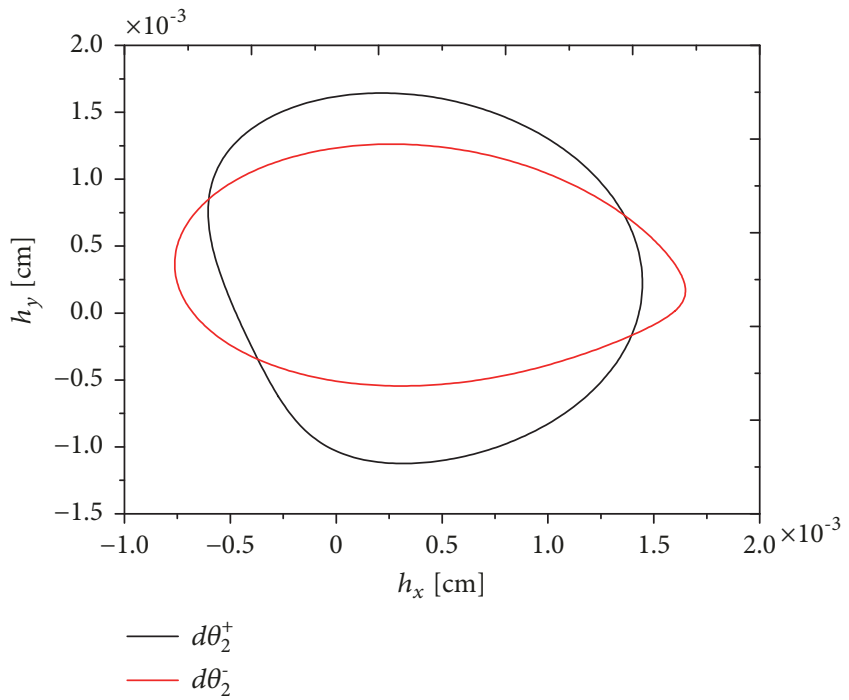

(b)

FIGURE 6: Error in $h$ from: (a) DLM. (b) RMH.

develop a similar trend between the deviations. However, if the prediction is compared with respect to the nominal position $p_{k}$, the deviations are maximum and minimum in some regions. For instance, it is denoted that in the range $\theta_{2} \in(2,5.4) \mathrm{rad}$ the errors in the output positions are minimized. It means that the prediction error was reduced $8 \%$ with respect to the maximum in this zone.

To observe the error accuracy, Figure 8(b) is computed taking the distances $\delta$ and $\beta$ that are related by (21). These parameters are described in Section 3. In practical terms, the distance $\delta$ shows the sensitivity of the output position with respect to the input angular variations $d \theta_{2}^{-}$and $d \theta_{2}^{+}$. When $\boldsymbol{\delta}$ takes minimum values, it means that in particular positions the mechanism is less sensitive to the input variations. For example in Figure 8(b), we determine the following intervals: $\theta_{2} \in(0.69,0.82)$ and $\theta_{2} \in(4.22,4.66) \mathrm{rad}$, which were established between peaks identified for each solution method (DLM and RMH). The extreme values of the intervals minimize the distance $\boldsymbol{\delta}$ that corresponds with the peaks; it means that $\boldsymbol{\delta}=0$. Consequently, these positions are favorable to describe the configuration of the mechanism without any influence of $d \theta_{2}^{ \pm}$. This information can be useful in the design 

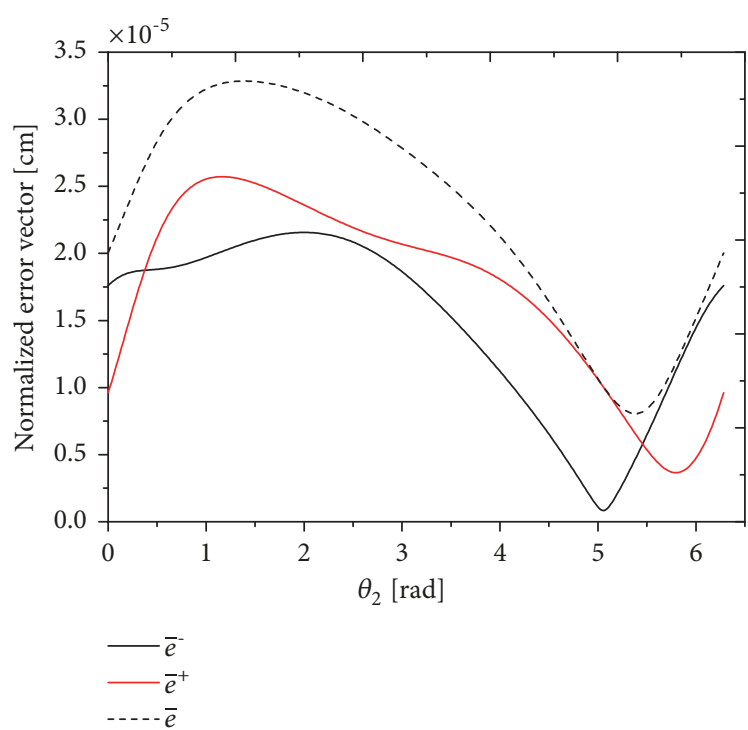

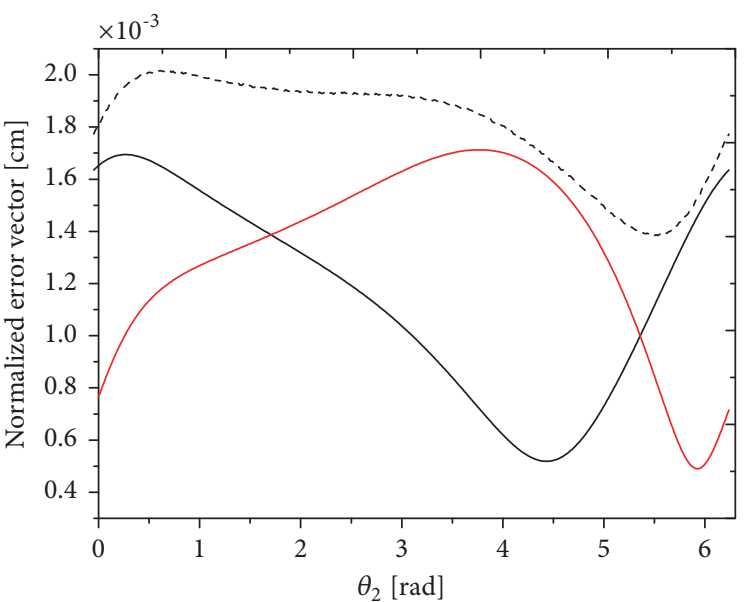

$\bar{e}^{-}$
$-\ldots \bar{e}$
$\bar{e}^{+}$

(a)

(b)

FiguRE 7: Absolute vector error: (a) DLM. (b) RMH.

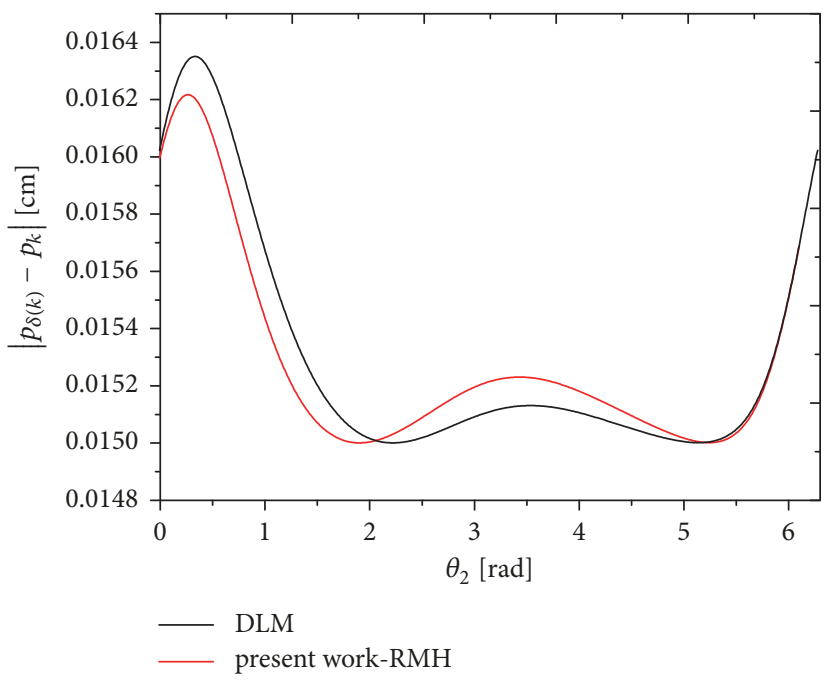

(a)

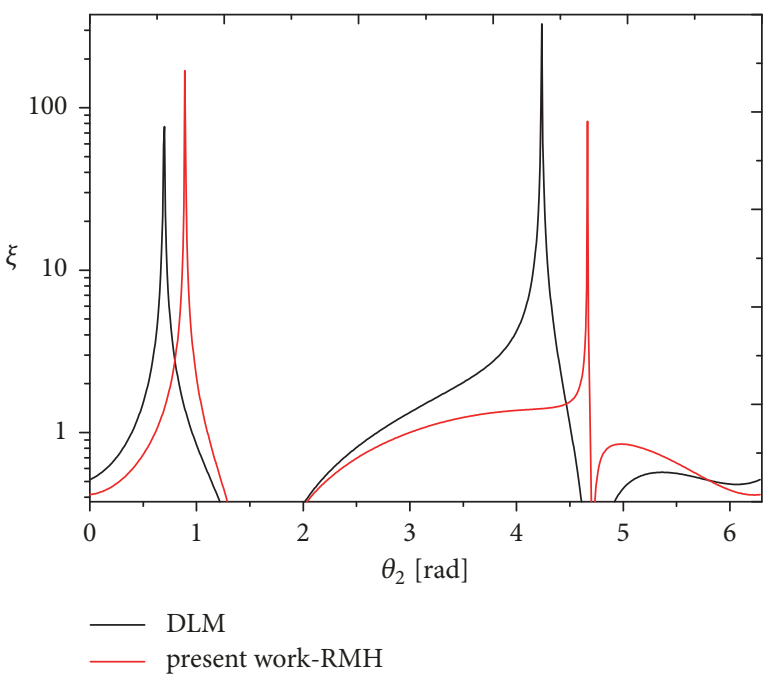

(b)

Figure 8: (a) Mean deviation for the output positions. (b) $r_{\max }$.

since we could predict where the mechanism is invariant to the input angular variations imposed by $d \theta_{2}^{ \pm}$.

\subsection{Estimation of a Local Error Domain by Input Angular} Variations $\left(d \theta_{2}^{-}\right.$and $\left.d \theta_{2}^{+}\right)$. In order to estimate a tolerance for the position of the point $A$, which is shown in Figure 4, a local domain error is predicted considering the method exposed in Section 3.2. Normally in a planar mechanism, the output position $\left(p_{k}\right)$ is estimated by means of a deterministic value when it does not present any type of variations (angular and dimensional). On the other hand, when there are geometric variations, output positions $\left(p_{k}^{+}, p_{k}^{-}\right.$, and $\left.p_{k}\right)$ can be delimited inside a probabilistic error space as mentioned by Luo et al.
[3], which considers that the dimensional variations (design variables) are fixed nominal values, and only it will be taken into account input angular variations established inside interval $d \theta_{2} \in\left(d \theta_{2}^{-}, d \theta_{2}^{+}\right)$as uncontrollable parameters Chen et al. [28]. For this reason, all possible solutions will only depend on input angular variations $d \theta_{2}$. The FBM used as example (see Section 4.1) will help to illustrate the exposed approach.

For the point $A$, a local space error was computed based on the solution obtained by RMH and DLM, but one of these solutions is shown in Figure 9. As explained before, for each position $k$ is determined a circular area $\Omega_{k}$ with center in a new predicted position $\bar{p}_{k}$ (see (20)) being the 


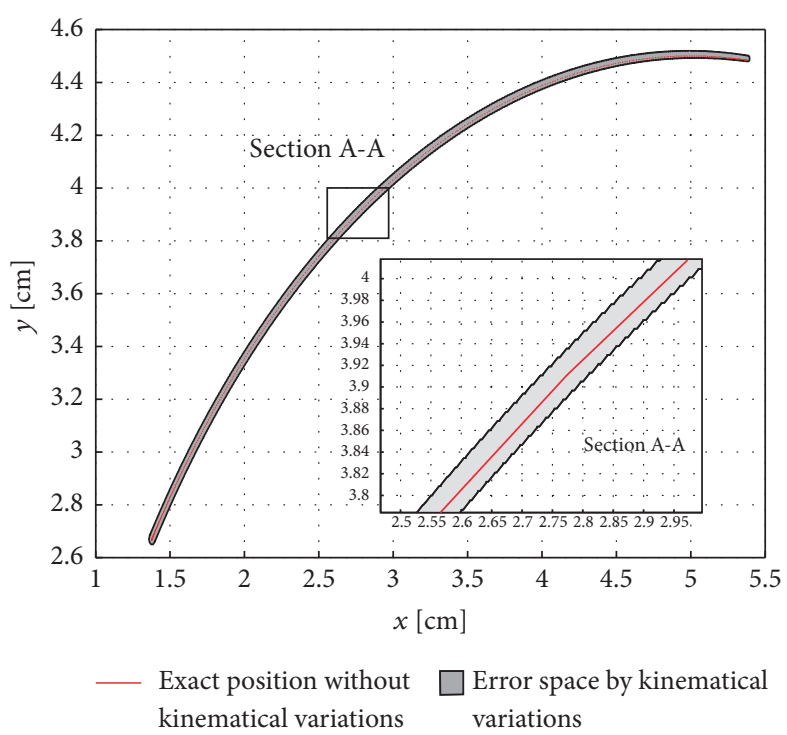

(a)

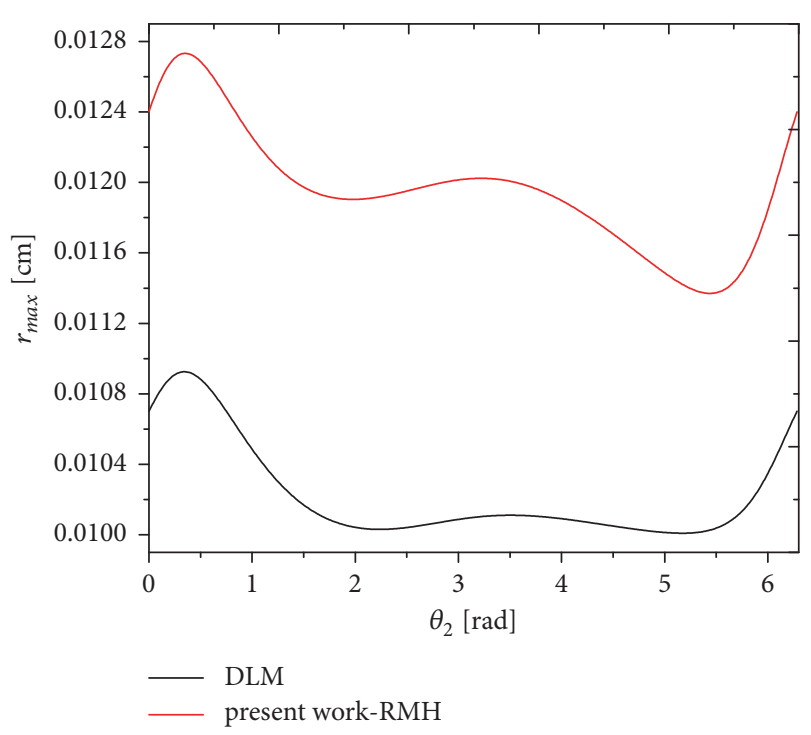

(b)

FIgURE 9: (a) Prediction of local error space at the point A (RMH method). (b) $r_{\max }$ comparisons.

total error domain delimited by the boundary of the set $C=\left\{\Omega_{1} \cup \Omega_{2} \cdots \Omega_{n}\right\}$, marked with gray color in the figure. The radius of $\Omega_{k}$ defines the tolerance of each output position. Tolerances are represented by $r_{\text {max }}$ defined in (20) and these are shown in Figure 9(b). It is observed that in some regions the radius is minimum and in others it is maximum. The results show that the tolerances presented comparable values to these illustrated in Figure 8(a). It is noted that $r_{\max }$ determined by DLM is greater than $r_{\text {max }}$ calculated by RMH, $20 \%$ approximately.

\subsection{Estimation of a Global Error Domain for All Combinations} of Variations. Section 3.3 deals with the prediction of a global error domain that represents the solutions related to all possible input combination of geometric variations. For this purpose, a multivariate normal random algorithm (MNRA) was used to validate the proposed method using random parameters Chase et al. [29]. MNRA generates random numbers from values defined with its standard deviations; in our case these were represented by the nominal values, and the standard deviations by the variations. This set of values was then used to calculate the output positions with all possible combinations of the input variations. For our example, there were generated $N=1000$ random sets and a SONY VAIO PC (M350 2,27 GHz i3 CPU, 8 GB RAM) was used in the Windows 7 environment for the computations. Applying both solution methods (DLM and $\mathrm{RMH}$ ), the generated data by MNRA were used to validate the predicted error domains as illustrated in Figure 10.

In Section 3.3 the definition of a global error domain was presented, which is sharply demarcated by an ellipse that represents the possible solutions generated with the combination of input variations $d r_{1} \in(-0.02,0.02), d r_{2} \in(-0.01,0.01)$, $d r_{3} \in(-0.02,0.02)$, and $d r_{4} \in(-0.015,0.015)$. In Figure 10 are presented three cases of solution in which the output positions were determined by MNRA, for $\theta=20^{\circ}, 150^{\circ}, 270^{\circ}$. Points blue and green indicate output positions for the input nominal variations $d \theta_{2}^{+}=0.017 \mathrm{rad}$ and $d \theta_{2}^{-}=$ -0.017 rad. Points cyan and magenta represent the solutions with the listed values in Table 1 . Points red and black describe the positions obtained with the eigenvalue problem explained in Section 2.4. The error domains predicted by DLM and RMH are depicted in Figures 10(a) and 10(b). It is seen that DLM domains are much smaller than RMH domains if these are compared the ellipse areas, but all predictions delimited a boundary that enclosed the majority of solutions. As a conclusion, it can be stated that the predictions determined with the proposed method simplified the variation analysis. Therefore, it could be used as a design tool of planar mechanisms that include geometric variations.

\section{Conclusions}

As main conclusion, we pointed out that H-Based Residual Method (RMH) was successfully applied and was demonstrated that it is a complementary solution of Direct Linearization Method (DLM). Both methodologies (RMH and DLM) were compared and the results showed that these are mathematically correlated. The reason is given by the nature of each solution since DLM minimizes a residual functional and $\mathrm{RMH}$ maximizes it, as explained theoretically in this study. Some differences were evidenced in the presented numerical example, for which was proposed a FBM. As relevant results were determined intervals where output positions were invariant with respect to the angular variations of the crank. DLM and RMH showed small differences between these. The computations were performed through a distance ratio established with the output deviations. In order to make the results more applied, error domains were 


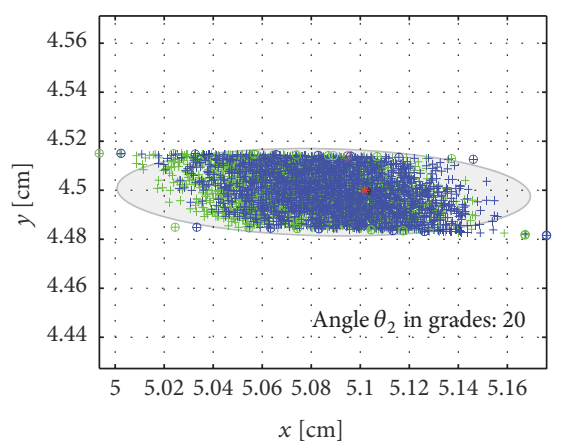

Nominal variations Combined variations

$\circ p_{k}^{+} \quad * p_{k}^{\lambda+} \quad \circ p_{k}^{+}$

$p_{k}^{-} \quad * p_{k}^{\lambda-} \quad \circ p_{k}^{-}$

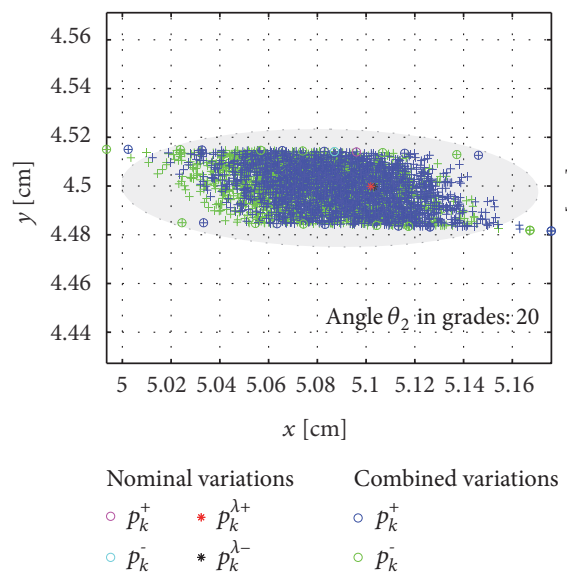

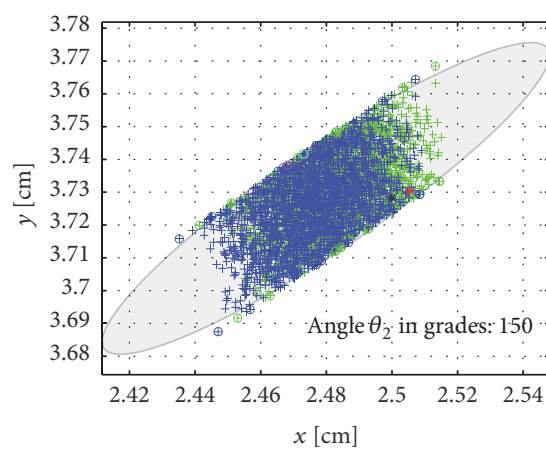

Nominal variations Combined variations

$\circ p_{k}^{+} \quad * p_{k}^{\lambda+} \quad \circ p_{k}^{+}$

$p_{k}^{-} \quad * p_{k}^{\lambda-}$

(a)

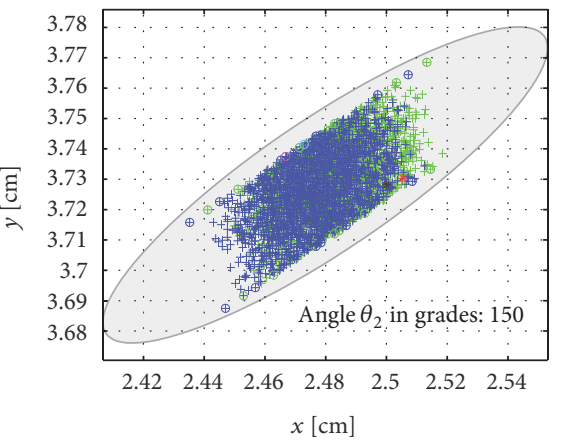

Nominal variations Combined variations $\circ p_{k}^{+} \quad * p_{k}^{\lambda+} \quad \circ p_{k}^{+}$

$p_{k}^{-} \quad * p_{k}^{\lambda-}$

(b)

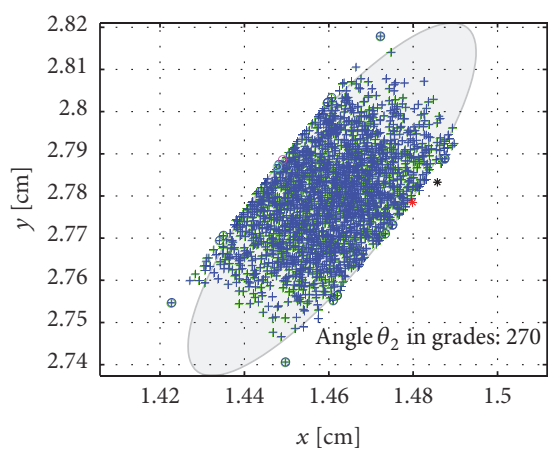

Nominal variations Combined variations

$\circ p_{k}^{+} \quad * p_{k}^{\lambda+} \quad \circ p_{k}^{+}$

$p_{k}^{-} \quad * p_{k}^{\lambda-} \quad \circ p_{k}^{-}$

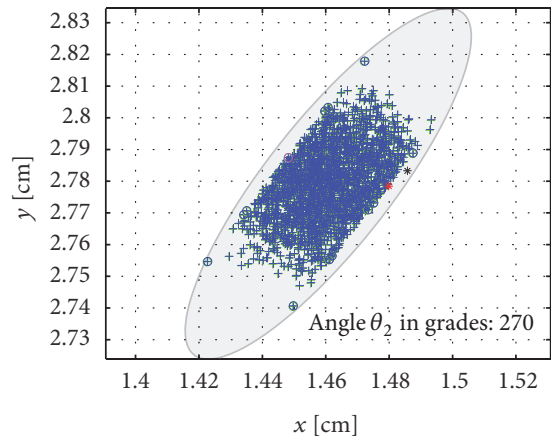

$$
\begin{array}{lll}
\text { Nominal variations } & \text { Combined variations } \\
\circ p_{k}^{+} & * p_{k}^{\lambda+} & \circ p_{k}^{+} \\
\circ p_{k}^{-} \quad * p_{k}^{\lambda-} & \circ p_{k}^{-}
\end{array}
$$

FIGURE 10: Global space domain for different positions of $\theta_{2}=20^{\circ}, 150^{\circ}, 270^{\circ}$. (a) DLM. (b) RMH.

calculated for predicting the output positions as geometric tolerances. It was proven by means of a variation analysis that all combinations of input variations generated by a multivariate normal random algorithm were delimited by the error domains predicted with RMH and DLM. However, the error domain determined with RMH presented a higher size than the domain calculated with DLM; it indicates that $\mathrm{RMH}$ is a better method to predict tolerances in the output positions.

\section{Data Availability}

The data used to support the findings of this study are available from the corresponding author upon request. However, these can be reproduced with the methodology exposed in the paper.

\section{Conflicts of Interest}

The authors declare that they have no conflicts of interest.

\section{Acknowledgments}

This research was funded by a project from Universidad Autónoma de Manizales with the project code 423-057 and obtained in the announcement of the year 2015.

\section{References}

[1] S. Khodaygan, "Manufacturing error compensation based on cutting tool location correction in machining processes," International Journal of Computer Integrated Manufacturing, vol. 27, no. 11, pp. 969-978, 2014.

[2] W. Wu and S. S. Rao, "Uncertainty analysis and allocation of joint tolerances in robot manipulators based on interval analysis," Reliability Engineering \& System Safety, vol. 92, no. 1, pp. 54-64, 2007.

[3] K. Luo and X. Du, "Probabilistic mechanism analysis with bounded random dimension variables," Mechanism and Machine Theory, vol. 60, pp. 112-121, 2013.

[4] P. Flores, "A methodology for quantifying the kinematic position errors due to manufacturing and assembly tolerances," 
Strojniški vestnik - Journal of Mechanical Engineering, vol. 57, no. 06, pp. 457-467, 2011.

[5] Y14.5-2018 ASME, "Dimensioning and tolerancing," in The American Society of Mechanical Engineers, ASME, New York, NY, USA, 2018

[6] 1101:2017 ISO, "Geometrical product specifications (gps, geometrical tolerancing, tolerances of form, orientation)," 2017.

[7] K. W. Chase and A. R. Parkinson, "A survey of research in the application of tolerance analysis to the design of mechanical assemblies," Research in Engineering Design, vol. 3, no. 1, pp. 2337, 1991.

[8] F. A. Lara-Molina, E. H. Koroishi, V. Steffen, and L. A. Martins, "Kinematic performance of planar 5R symmetrical parallel mechanism subjected to clearances and uncertainties," Journal of the Brazilian Society of Mechanical Sciences and Engineering, vol. 40 , no. 4 , article $189,2018$.

[9] H. P. Jawale and A. Jaiswal, "Investigation of mechanical error in four-bar mechanism under the effects of link tolerance," Journal of the Brazilian Society of Mechanical Sciences and Engineering, vol. 40, no. 8, article 383, 2018.

[10] A. Hofmeister, W. Sextro, and O. Röschel, "Error workspace analysis of planar mechanisms," in EUCOMES, the first European Conference on Mechanism Science, Obergurgl, Austria, 2006.

[11] J. D. Marler, Nonlinear tolerance analysis using the direct linearization method [Ph.D. thesis], Brigham Young University, Department of Mechanical Engineering, 1988.

[12] J. W. Wittwer, K. W. Chase, and L. L. Howell, "The direct linearization method applied to position error in kinematic linkages," Mechanism and Machine Theory, vol. 39, no. 7, pp. 681693, 2004.

[13] B. M. Imani and M. Pour, "Tolerance analysis of flexible kinematic mechanism using DLM method," Mechanism and Machine Theory, vol. 44, no. 2, pp. 445-456, 2009.

[14] R. C. Leishman and K. W. Chase, "Direct linearization method kinematic variation analysis," Journal of Mechanical Design, vol. 132, no. 7, Article ID 071003, 2010.

[15] S. K. Kim, S. S. Kim, Y. G. Cho, and H. K. Jung, "Accumulated tolerance analysis of suspension by geometric tolerances based on multibody elasto-kinematic analysis," International Journal of Automotive Technology, vol. 17, no. 2, pp. 255-263, 2016.

[16] L. Wang, X. Zhang, and Y. Zhou, "An effective approach for kinematic reliability analysis of steering mechanisms," Reliability Engineering \& System Safety, vol. 180, pp. 62-76, 2018.

[17] F. Malvezzi and T. A. Coelho, "Error analysis for an active geometry control suspension system," Journal of the Brazilian Society of Mechanical Sciences and Engineering, vol. 40, no. 12, article 558, 2018.

[18] A. Armillotta, "Tolerance analysis of gear trains by static analogy," Mechanism and Machine Theory, vol. 135, pp. 65-80, 2019.

[19] K.-S. Lin, K.-Y. Chan, and J.-J. Lee, "Kinematic error analysis and tolerance allocation of cycloidal gear reducers," Mechanism and Machine Theory, vol. 124, pp. 73-91, 2018.

[20] J. Rameau, P. Serré, and M. Moinet, "Clearance vs. tolerance for mobile overconstrained mechanisms," Mechanism and Machine Theory, vol. 136, pp. 284-306, 2019.

[21] Q. Zhao, J. Guo, and J. Hong, "Assembly precision prediction for planar closed-loop mechanism in view of joint clearance and redundant constraint," Journal of Mechanical Science and Technology, vol. 32, no. 7, pp. 3395-3405, 2018.
[22] J. Fan, H. Tao, C. Wu, R. Pan, Y. Tang, and Z. Li, "Kinematic errors prediction for multi-axis machine tools guideways based on tolerance," The International Journal of Advanced Manufacturing Technology, vol. 98, no. 5-8, pp. 1131-1144, 2018.

[23] Y. Ni, C. Shao, B. Zhang, and W. Guo, "Error modeling and tolerance design of a parallel manipulator with full-circle rotation," Advances in Mechanical Engineering, vol. 8, no. 5, pp. 1-16, 2016.

[24] R. L. Norton, Design of Machinery: An Introduction to the Synthesis and Analysis of Mechanisms and Machines, McGrawHill, Boston, Mass, USA, 2nd edition, 1999.

[25] H. A. Tinoco and M. A. Flórez, "A new method for determining position errors of planar mechanisms including dimensional variations in its linkages," in Proceedings of the XII PanAmerican Congress of Applied Mechanics (PACAM XII), 2012.

[26] J. Gao, K. W. Chase, and S. P. Magleby, "Generalized 3-d tolerance analysis of mechanical assemblies with small kinematic adjustments," IIE transactions, vol. 30, no. 4, pp. 367-377, 1998.

[27] S. Rajagopalan and M. Cutkosky, "Error analysis for the in-situ fabrication of mechanisms," Journal of Mechanical Design, vol. 125, no. 4, pp. 809-822, 2003.

[28] W. Chen, J. K. Allen, K.-L. Tsui, and F. Mistree, "A procedure for robust design: Minimizing variations caused by noise factors and control factors," Journal of Mechanical Design, vol. 118, no. 4, pp. 478-485, 1996.

[29] K. W. Chase, J. Gao, and S. P. Magleby, "General 2-d tolerance analysis of mechanical assemblies with small kinematic adjustments," Journal of Design and Manufacturing, vol. 5, pp. 263274, 1995. 


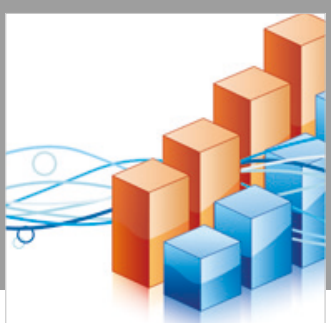

Advances in

Operations Research

\section{-n-m}
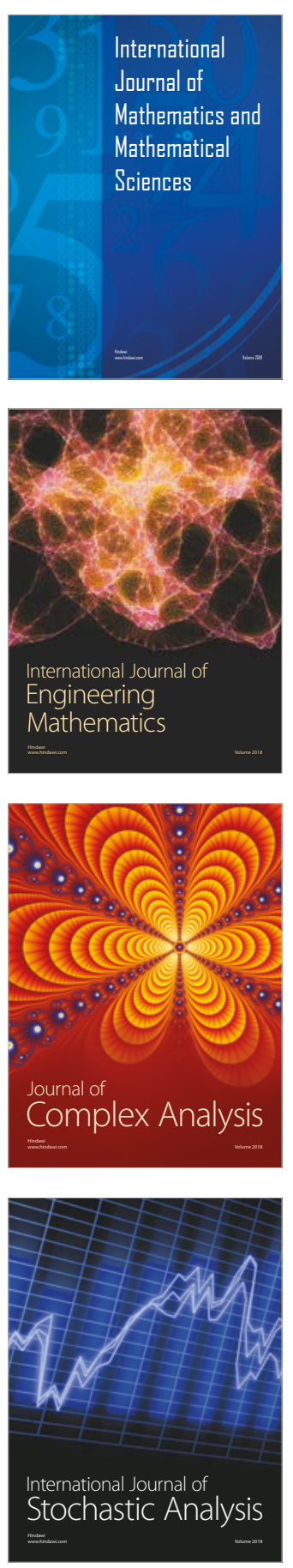
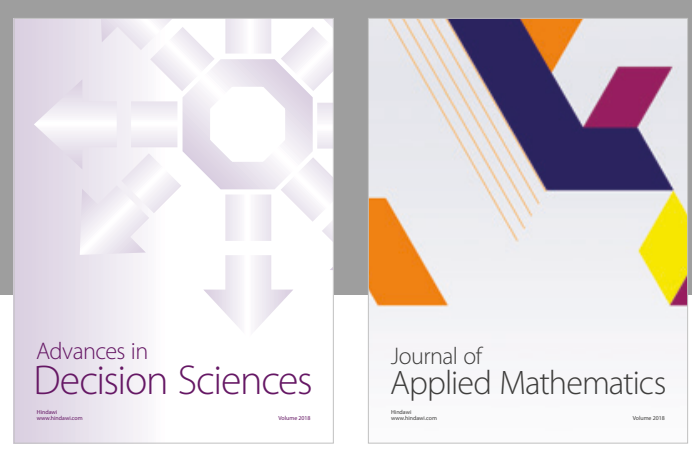

Journal of

Applied Mathematics
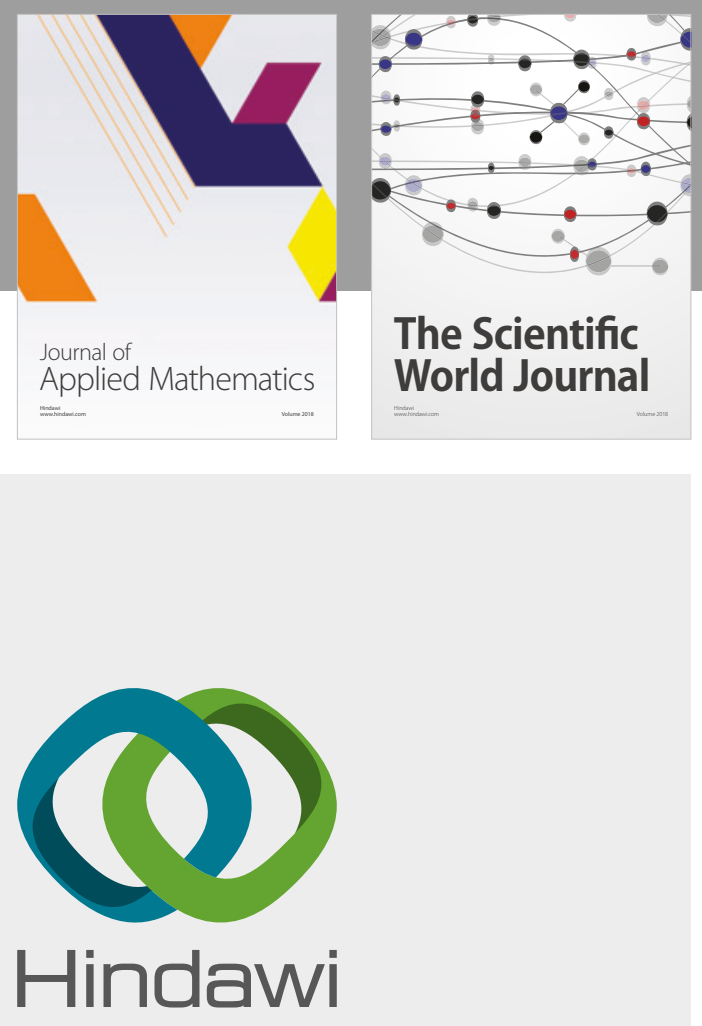

Submit your manuscripts at

www.hindawi.com

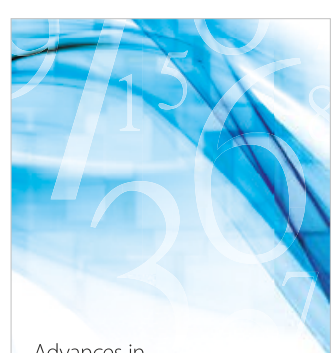

Advances in
Numerical Analysis
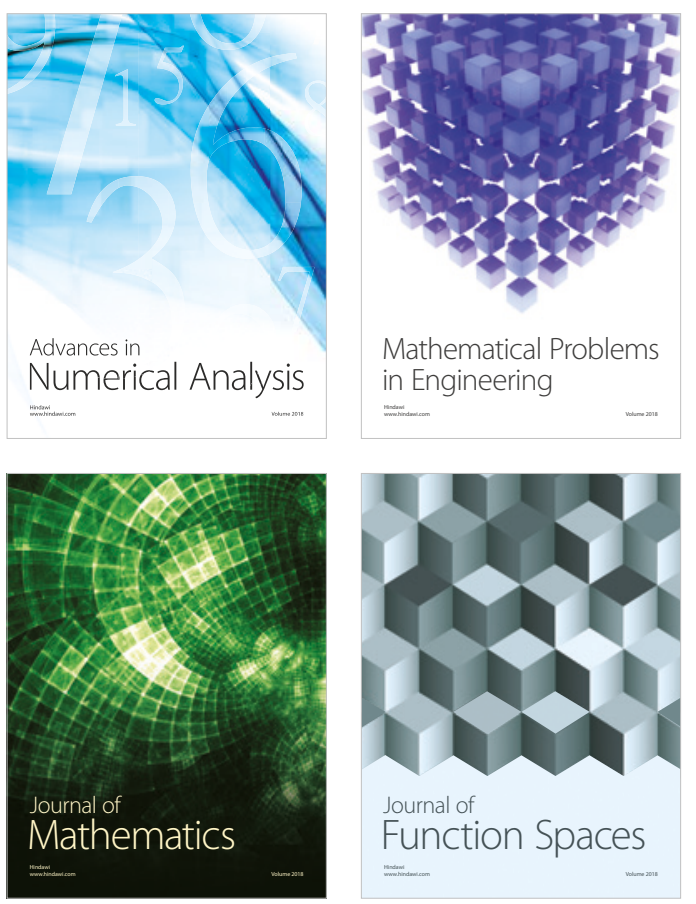

Mathematical Problems in Engineering

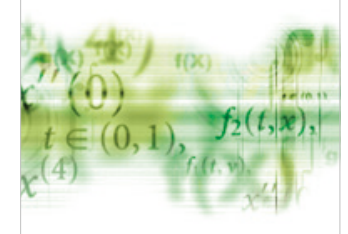

International Journal of

Differential Equations

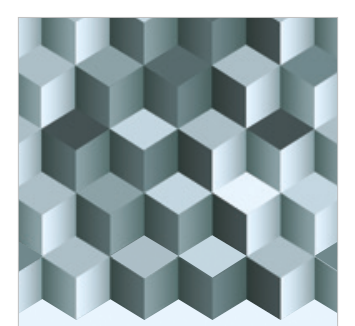

Journal of

Function Spaces

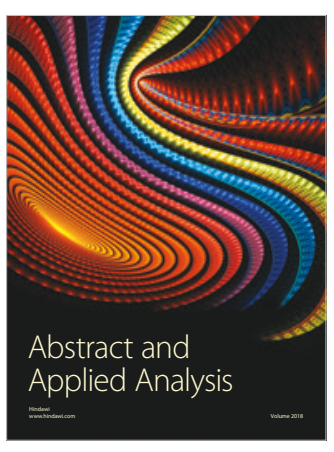

The Scientific

World Journal

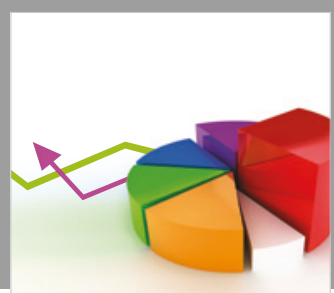

Journal of

Probability and Statistics
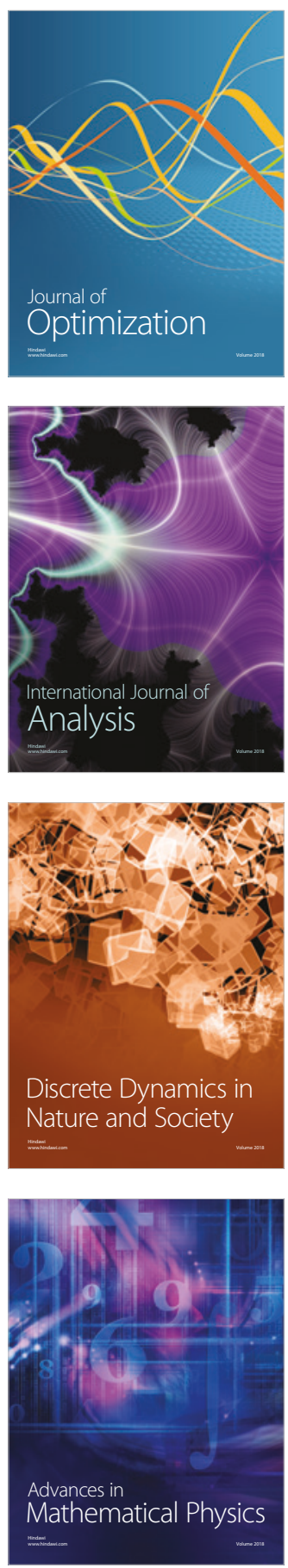